Zbl Arbeitsmed 2020 $\cdot 70: 57-65$ https://doi.org/10.1007/s40664-019-00373-7 Online publiziert: 19. September 2019 (c) Der/die Autor(en) 2019

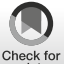

Anika D. Schulz ${ }^{1}$ Johannes Wendsche ${ }^{2}$. Andrea Lohmann-Haislah ${ }^{3}$. Ina Schöllgen'

'FB 3 Arbeit und Gesundheit, Gruppe 3.4 Mentale Gesundheit und kognitive Leistungsfähigkeit, Bundesanstalt für Arbeitsschutz und Arbeitsmedizin, Berlin, Deutschland

${ }^{2}$ FB 3 Arbeit und Gesundheit, Gruppe 3.6 Betriebliche Intervention und Transfer, Bundesanstalt für Arbeitsschutz und Arbeitsmedizin (BAuA), Dresden, Deutschland

${ }^{3} \mathrm{FB} 3$ Arbeit und Gesundheit, Gruppe 3.5 Psychische Belastungen, Bundesanstalt für Arbeitsschutz und Arbeitsmedizin (BAuA), Berlin, Deutschland

\title{
Erholungsbeeinträchtigungen bei Beschäftigten
}

\section{Ergebnisse einer Repräsentativbefragung in Deutschland}

bestehenden Erholungsproblemen langfristig akkumulieren kann. Die Folge sind chronische körperliche und mentale Gesundheitsbeeinträchtigungen [3]. Demzufolge wird eine beeinträchtigte Erholung auch als Frühindikator für die Entwicklung drohender Gesundheitseinschränkungen betrachtet [9].

Bisher ist nicht geklärt, wie stark Erholungsschwierigkeiten bei Beschäftigten in Deutschland verbreitet sind und ob bestimmte Beschäftigtengruppen besonders betroffen sind. In diesem Beitrag wird deshalb überblicksartig die Prävalenz von Erholungsbeeinträchtigungen bei Beschäftigten anhand repräsentativer Daten vorgestellt. Hierbei werden auch bestimmte als abträglich für die Erholung diskutierte Beschäftigungsmerkmale berücksichtigt [17]. Außerdem wird untersucht, ob Personen spezifischer Berufsgruppen stärker mit Erholungsproblemen konfrontiert sind und welche Zusammenhänge mit der Arbeitsfähigkeit und der mentalen Gesundheit von Beschäftigten existieren.

\section{Methodik}

\section{Stichprobe}

Die hier vorgestellten Auswertungen beruhen auf Daten der ersten Erhebungswelle der Studie zur Mentalen Gesundheit bei der Arbeit (S-MGA). Es han- delt sich dabei um eine Repräsentativbefragung sozialversicherungspflichtig Beschäftigter im Alter von 31 bis 60 Jahren in Deutschland. Die Studie wurde von der Bundesanstalt für Arbeitsschutz und Arbeitsmedizin veranlasst und zusammen mit dem Institut für Arbeitsmarkt- und Berufsforschung sowie dem infas Institut für angewandte Sozialwissenschaft $\mathrm{GmbH}$ von November 2011 bis Juni 2012 durchgeführt. Hierbei wurden 4511 Erwerbstätige befragt. Die Stichprobe beinhaltet Vollzeit- und Teilzeitbeschäftigte sowie Mini- und Midi-Jobber. Da sich die hier untersuchten Fragestellungen stets auf den Arbeitskontext beziehen, wurden für die Analysen Personen ausgeschlossen, deren Status sich in der Zeit zwischen der Stichprobenrekrutierung und der Datenerhebung auf arbeitslos, arbeitssuchend, längere Krankmeldung oder Ruhestand geändert hatte. Damit beläuft sich die den Auswertungen zugrunde liegende Stichprobe auf 4201 Befragte. Das Geschlechterverhältnis war hierbei nahezu ausgeglichen (2105 Frauen, 2096 Männer). Weitere Details zum Studiendesign und zur Stichprobenziehung der S-MGA sind dem dazugehörigen Kohortenprofil zu entnehmen [12].

\section{Datenerhebung}

Die S-MGA wurde mithilfe von computergestützten persönlichen Interviews dung einher, die sich jedoch bei länger 
Tab. 1 Erholungsbeeinträchtigungen nach Alter und Geschlecht

\begin{tabular}{|c|c|c|c|c|c|c|c|c|c|c|}
\hline & \multirow[t]{2}{*}{ Altersspanne } & \multicolumn{3}{|c|}{$\begin{array}{l}\text { Unauffälliges Erholungserleben } \\
<75 \% \text {-Perzentil der Vergleichs- } \\
\text { gruppe }\end{array}$} & \multicolumn{3}{|c|}{$\begin{array}{l}\text { Auffällige Erholungsunfähigkeit } \\
75 \text { - bis } 90 \% \text {-Perzentil der Ver- } \\
\text { gleichsgruppe }\end{array}$} & \multicolumn{3}{|c|}{$\begin{array}{l}\text { Sehr auffällige Erholungsunfähigkeit } \\
>90 \% \text {-Perzentil der Vergleichsgrup- } \\
\text { pe }\end{array}$} \\
\hline & & $N$ & $\%$ & $95 \%-K I$ & $N$ & $\%$ & $95 \%-K I$ & $N$ & $\%$ & $95 \%-K I$ \\
\hline \multirow[t]{4}{*}{ Männer } & 31 bis 40 Jahre & 473 & 87 & $83-89$ & 33 & 6 & $4-8$ & 42 & 8 & $5-10$ \\
\hline & 41 bis 50 Jahre & 739 & 86 & $84-88$ & 56 & 6 & $5-8$ & 61 & 7 & $6-9$ \\
\hline & 51 bis 60 Jahre & 590 & 87 & $84-89$ & 43 & 6 & $5-8$ & 48 & 7 & $5-9$ \\
\hline & Insgesamt & 1802 & 87 & $85-88$ & 132 & 6 & $5-7$ & 151 & 7 & $6-8$ \\
\hline \multirow[t]{4}{*}{ Frauen } & 31 bis 40 Jahre & 391 & 84 & $81-88$ & 30 & 7 & $5-10$ & 42 & 9 & $6-12$ \\
\hline & 41 bis 50 Jahre & 772 & 86 & $84-89$ & 57 & 7 & $5-9$ & 62 & 7 & $5-9$ \\
\hline & 51 bis 60 Jahre & 643 & 87 & $84-89$ & 41 & 6 & $4-8$ & 55 & 7 & $6-10$ \\
\hline & Insgesamt & 1806 & 86 & $84-88$ & 128 & 6 & $5-8$ & 159 & 7 & $6-9$ \\
\hline
\end{tabular}

(CAPI) unter Verwendung wissenschaftlich etablierter Instrumente durchgeführt. Während der Befragung legten die Interviewer zur Unterstützung Listen vor, in dem die Antwortkategorien für die gestellten Fragen aufgeführt waren. Die jeweiligen Antworten der Befragten wurden von Interviewern im Anschluss elektronisch erfasst. Die momentane psychosoziale Situation der Studienteilnehmer wurde hingegen schriftlich abgefragt.

\section{Instrumente}

\section{Erholungsbeeinträchtigungen}

Das Ausmaß der Erholungsbeeinträchtigungen wurde mithilfe der Dimension Erholungsunfähigkeit aus dem Fragebogen zur Analyse belastungsrelevanter Aufgabenbewältigung [11] ermittelt. Die Befragten gaben hierfür den Grad ihrer Zustimmung zu 6 Aussagen auf einer 4-stufigen Ratingskala von 1 (trifft überhaupt nicht zu) bis 4 (trifft sehr zu) an. Höhere Werte stehen für stärker ausgeprägte Erholungsprobleme. Eine Beispielaussage lautet: „Es fällt mir schwer, nach der Arbeit abzuschalten“. Alle Aussagen weisen dabei stets einen Arbeitsbezug auf, und es werden Verhaltensweisen abgefragt, die bei Belastungssituationen mit dauerhaften Aktivierungen einhergehen. Unter anderem werden auch arbeitsbedingte Schlafstörungen und das gedankliche Beschäftigen mit Arbeitsproblemen im Urlaub erfragt. Die Erfassung der Antworten erfolgte mittels CAPI. Zur Auswertung wurden alle 6 Ant- worten zu einem Punktsummenwert aufaddiert und folglich nur Personen eingeschlossen, die eine Einschätzung für jede Aussage abgegeben hatten. Mithilfe vorliegender Normwerte wurden die Befragten 3 Gruppen zugeordnet, die sich in ihrem Grad der Erholungsbeeinträchtigung unterscheiden [10]. Studienteilnehmer mit einem Summenwert bis 18 (75\%-Perzentil) wurden als unauffällig, von 19 bis 20 als auffällig und ab 21 (90\%Perzentil) als sehr auffällig eingestuft.

\section{Beschäftigungsmerkmale}

Im Rahmen des CAPI wurden außerdem Angaben $\mathrm{zu}$ verschiedenen Beschäftigungsmerkmalen der Befragten erfasst. Hierzu gehörten die gewöhnliche Anzahl an Arbeitsstunden pro Woche (einschließlich regelmäßig geleisteter Überstunden, Mehrarbeit etc.), die Lage der Arbeitszeit (Zeiten für Arbeitsbeginn und -ende, Gleitzeit, Schichtdienst etc.) sowie die Art des derzeitigen Arbeitsvertrags (zeitlich befristet, unbefristet).

\section{Berufsgruppen}

Die aktuelle berufliche Tätigkeit der Beschäftigten wurde im CAPI mittels offener Nennungen der Befragten dokumentiert. Diese Angaben wurden anschließend durch geschulte Kodierer in Berufsgruppen gemäß der International Standard Classification of Occupations 2008 [6] überführt. Für die vorliegenden Auswertungen erfolgte eine Zusammenfassung zu insgesamt 12 Gruppen.

\section{Arbeitsfähigkeit}

Zur Ermittlung der aktuellen Arbeitsfähigkeit der Befragten wurde der Work Ability Index $[15,16]$ verwendet. Er beinhaltet insgesamt 10 Fragen zu den körperlichen und geistigen Arbeitsanforderungen, zum Gesundheitszustand sowie $\mathrm{zu}$ den Leistungsreserven der befragten Erwerbstätigen. Im Gesamtergebnis wurde verfahrensgemäß aus diesen Angaben ein Punktsummenwert zwischen 7 und 49 Punkten ermittelt, der Auskunft über die momentane Fähigkeit gibt, vorhandene Arbeitsanforderungen zu bewältigen. Eine verminderte Arbeitsfähigkeit wurde mit einem Punktwert zwischen 7 und 27 definiert [4]. Befragte mit unvollständigen Angaben für dieses Instrument wurden aus den Auswertungen ausgeschlossen.

\section{Mentale Gesundheit}

Die mentale Gesundheit der Befragten wurde durch das Ausmaß der Erschöpfung und der depressiven Symptomatik abgebildet.

Die selbst eingeschätzte Erschöpfung wurde schriftlich mit dem Oldenburg Burnout Inventar [1, 2] ermittelt. Auf einer 4-stufigen Skala von 1 (völlig unzutreffend) bis 4 (völlig zutreffend) gaben die Befragten jeweils an, in welchem Umfang sie insgesamt 8 Aussagen zustimmten. Eine Beispielaussage lautet: „Es gibt Tage, an denen ich mich schon vor der Arbeit müde fühle“. Invers formulierte Aussagen wurden rekodiert, sodass hohe Werte stets ein stärkeres Ausmaß der Erschöpfung darstellen. Aus den 8 Antwor- 
Zbl Arbeitsmed 2020 · 70:57-65 https://doi.org/10.1007/s40664-019-00373-7

(c) Der/die Autor(en) 2019

\section{A. D. Schulz · J. Wendsche · A. Lohmann-Haislah • I. Schöllgen}

\section{Erholungsbeeinträchtigungen bei Beschäftigten. Ergebnisse einer Repräsentativbefragung in Deutschland}

\section{Zusammenfassung}

Hintergrund. Das Thema Erholung von der Arbeit gewinnt in der Forschung und in der betrieblichen Praxis zunehmend an Bedeutung. Auftretende Schwierigkeiten, sich zu erholen, können ein frühes Warnsignal für drohende Gesundheitseinschränkungen sein. Ziel der Arbeit. Die vorliegende Untersuchung gibt einen Überblick über die Verbreitung von Erholungsbeeinträchtigungen bei Beschäftigten in Deutschland. Dabei werden verschiedene Beschäftigungsmerkmale und Berufsgruppen berücksichtigt. Außerdem werden Erholungsprobleme in Verbindung mit gesundheitsbezogenen Aspekten wie Arbeitsfähigkeit, Erschöpfung und depressiven Symptomen betrachtet.
Methodik. Es wurden repräsentative Daten von 4201 sozialversicherungspflichtig Beschäftigten in Deutschland aus der ersten Erhebungswelle der Studie Mentale Gesundheit bei der Arbeit (S-MGA) ausgewertet.

Ergebnisse. Die Ergebnisse zeigen, dass 13\% der Befragten in ihrer Erholung beeinträchtigt sind. Personen mit einer hohen Anzahl an Wochenarbeitsstunden, ungeregelten Arbeitszeiten und/oder befristeten Verträgen sind anteilig am meisten von Erholungsproblemen betroffen. Erholungsschwierigkeiten treten prozentual am häufigsten bei Führungskräften und Beschäftigten mit Interaktionsarbeit auf. Mit steigendem Grad der Erholungsbeeinträchtigungen nimmt auch gleichzeitig der jeweilige Anteil an Beschäftigten mit Erschöpfung, depressiven Symptomen sowie verminderter Arbeitsfähigkeit zu.

Diskussion. Die Ergebnisse liefern Anhaltspunkte zur Identifikation beruflicher Risikogruppen für potenzielle Erholungsprobleme. Diese können mit reduzierter Arbeitsfähigkeit und eingeschränkter mentaler Gesundheit einhergehen. Im Rahmen der Gefährdungsbeurteilung sollten folglich auch die Erholungsbedingungen während und nach der Arbeit in den Blick genommen werden.

Schlüsselwörter

Erholung · Arbeitsfähigkeit · Mentale Gesundheit · Erschöpfung · Depressivität

\section{Impaired recovery among employees. Results from a representative survey in Germany}

\section{Abstract}

Background. In recent years, the subject of recovery from work has become increasingly important in research and at the workplace. Experiencing difficulty with properly recovering from work can be an early warning sign for potential deterioration in health. Objective. The present study seeks to provide an overview on the prevalence of experiencing difficulty with recovering from work among employees in Germany. In addition, impaired recovery from work was examined across different occupational groups and how this relates to various employment characteristics and health-related issues, such as work ability, exhaustion and depressive symptoms.

\begin{abstract}
Material and methods. Analyses were carried out on representative data from the first wave of the study on mental health at work (S-MGA) consisting of 4201 employees who are legally required to make social security contributions in Germany.

Results. The findings reveal that $13 \%$ of the study participants experience difficulty with recovering from work. Employees who have long or irregular working hours and those with fixed-term contracts are the most impaired in their ability to recover from work. Difficulty with recovering from work most often occurs in employees in managerial or supervisory positions and in occupations with interactive work. As difficulty with recovering increases,
\end{abstract}

the proportion of employees with symptoms of exhaustion, depressive symptoms and reduced work ability also rises.

Conclusion. This analysis provides initial evidence for identifying occupational groups at risk for experiencing difficulty with recovering from work. Such experiences can be associated with reduced work ability and impaired mental health. Therefore, the conditions for recovery during and after work should be taken into account within the scope of risk assessments.

\section{Keywords}

Recovery · Work ability - Mental health . Exhaustion · Depressive symptoms ten wurde ein Durchschnittswert gebildet, wobei das Vorliegen einer Erschöpfung mit einem Schwellenwert von $\geq 3$ für die vorliegende Ergebnisauswertung definiert wurde. Personen mit fehlenden Werten $\mathrm{zu}$ diesem Instrument wurden aus den Analysen ausgeschlossen.

Um die Schwere einer potenziellen depressiven Symptomatik zu erfassen, wurde ebenfalls schriftlich das Depressionsmodul des Gesundheitsfragebogens für Patienten eingesetzt. Es handelt sich dabei um die deutsche Version des PRI-
ME MD Patient Health Questionnaire [5, 14]. Die Beschäftigten wurden gebeten einzuschätzen, wie häufig sie sich in den letzten 2 Wochen durch 9 verschiedene Beschwerden beeinträchtigt fühlten, wie z. B. Niedergeschlagenheit, Schwermut oder Hoffnungslosigkeit. Die Antworten wurden mithilfe einer 4-stufigen Ratingskala gegeben, die von 0 (überhaupt nicht) bis 3 (beinahe jeden Tag) reicht. Aus den 9 Antworten wurde ein Summenwert gebildet, der zwischen 0 und 27 liegt. Gemäß den
Auswertungsempfehlungen für dieses Instrument wurde ein Summenwert von $\geq 10$ als Hinweis auf das Vorliegen einer depressiven Symptomatik eingestuft. In die Summenwertbildung sind nur Personen eingeflossen, die alle 9 Aussagen beantwortet hatten.

\section{Datenauswertung}

Die hier vorgestellten Berechnungen wurden mithilfe der Statistiksoftware IBM SPSS Statistics (Version 25) durch- 


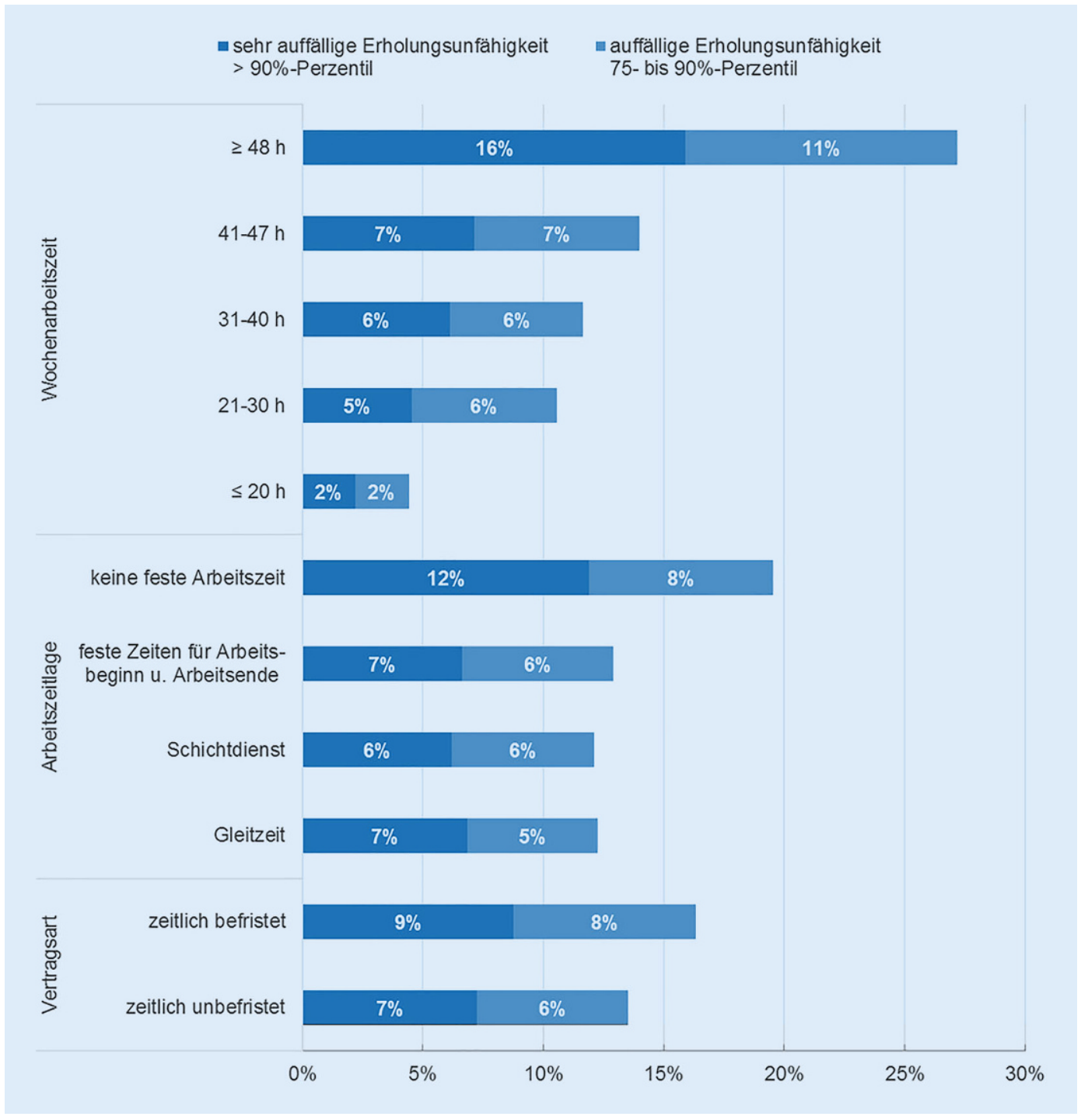

Abb. $1<$ Prozent der Beschäftigten getrennt nach Beschäftigungsmerkmalen mit auffälligen und sehr auffälligen Einschränkungen in der Erholung geführt. Die Datenauswertung erfolgte stets mit gewichteten Stichprobenangaben, um leichte Abweichungen von der zugrunde liegenden Grundgesamtheit der in Deutschland sozialversicherungspflichtig Beschäftigten im genannten Altersbereich auszugleichen [12].

\section{Ergebnisse}

\section{Prävalenz von Erholungs- beeinträchtigungen}

Die Ergebnisse zeigen, dass $6 \%$ der Beschäftigten auffällige Einschränkungen im Erholungserleben aufweisen. Ihre Werte liegen entsprechend zwischen dem $75 \%$ - und dem $90 \%$-Perzentil der Verteilung der Normstichprobe. Zusätzliche $7 \%$ der Studienteilnehmer fallen in die Kategorie mit sehr auffälligen Erholungsschwierigkeiten und erreichen damit Werte über dem $90 \%$-Perzentil.

Bei einer geschlechterspezifischen Betrachtung der in der Stichprobe ermittelten Erholungsschwierigkeiten finden sich ähnliche Werte bei Frauen und Männern (• Tab. 1). Auch die getrennte Betrachtung verschiedener Altersgruppen deutet auf ähnliche prozentuale Anteile an Personen mit auffälligen und sehr auffälligen Schwierigkeiten beim Erholungserleben in den unterschiedlichen Altersgruppen hin.

\section{Beschäftigungsmerkmale und Erholung}

In Abb. 1 sind die ermittelten $\mathrm{Zu}$ sammenhänge zwischen berichteten
Erholungsbeeinträchtigungen und den betrachteten Beschäftigungsmerkmalen abgebildet. Bezüglich der gewöhnlichen Wochenarbeitszeit zeigt sich, dass mit zunehmender Länge der tatsächlichen Wochenarbeitszeit der prozentuale Anteil an Beschäftigten mit auffälligen und sehr auffälligen Erholungsproblemen ansteigt. So leiden z. B. von den Beschäftigten mit einer typischen Arbeitszeit unter $20 \mathrm{~h}$ pro Woche insgesamt $4 \%$ unter auffälligen und sehr auffälligen Erholungsbeeinträchtigungen. Betrachtet man hingegen diejenigen mit den längsten Arbeitszeiten von 48 Arbeitsstunden und mehr pro Woche, so finden sich in dieser Gruppe mit insgesamt $27 \%$ anteilig die meisten Personen mit Erholungsproblemen. 


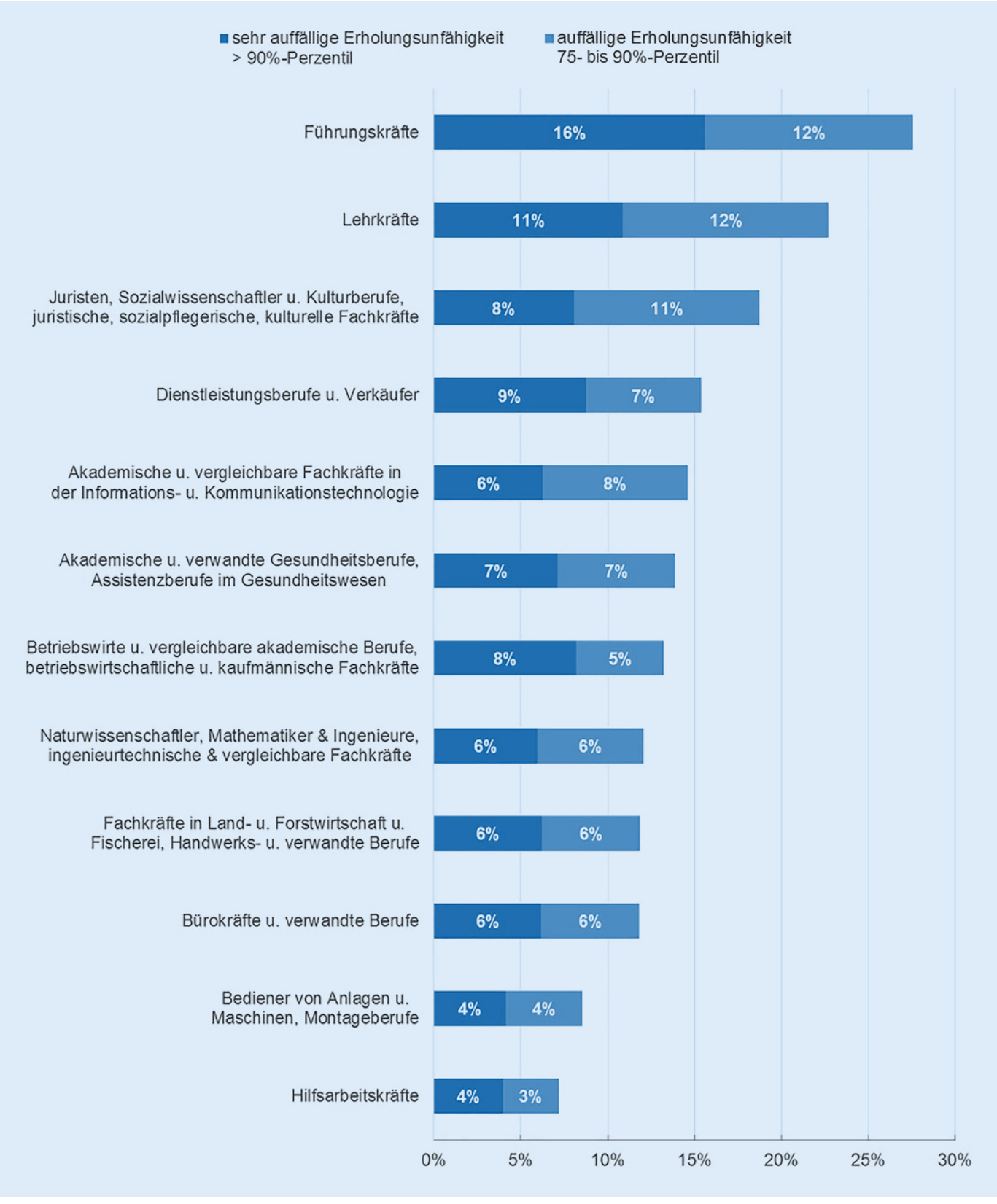

Abb. $2<$ Prozent der Beschäftigten je Berufsgruppe mit auffälligen und sehr auffälligen Einschränkungen in der Erholung
Hinsichtlich der Lage der Arbeitszeit befinden sich unter denjenigen ohne feste Arbeitszeiten mit insgesamt $20 \%$ die meisten Beschäftigten mit Erholungsbeeinträchtigungen. Anteilig weniger betroffen sind Befragte mit Gleitzeitregelung (12\%), festen Zeiten für Arbeitsbeginn und Arbeitsende (13\%) und Schichtdienst (12\%).

Bezüglich der berichteten Vertragsart sind in der Gruppe der befristet Beschäftigten insgesamt $17 \%$ von auffälligen und sehr auffälligen Erholungsproblemen betroffen (- Abb. 1). Im Vergleich dazu sind es bei Beschäftigten mit un- befristeten Verträgen insgesamt $13 \%$ mit auffällig und stark auffällig beeinträchtigter Erholung. Eine ausführliche Tabelle der berechneten Werte befindet sich im Anhang (•Tab. 2).

\section{Erholung bei verschiedenen Berufsgruppen}

In - Abb. 2 sind jeweils die prozentualen Anteile an Personen verschiedener Berufsgruppen mit auffälligen und sehr auffälligen Erholungsproblemen abgebildet. Die vorliegenden Auswertungen zeigen, dass Führungskräfte mit ins- gesamt $28 \%$ anteilig am meisten von Erholungsproblemen betroffen sind. Hierzu zählen unter anderem Geschäftsführer, leitende Verwaltungsbedienstete sowie Führungskräfte im kaufmännischen und Dienstleistungsbereich sowie in der Produktion und im Handel. Auch Berufsgruppen mit starkem Kunden-, Klienten- oder Patientenkontakt leiden eher unter Erholungsunfähigkeit. Zum Beispiel liegen 12\% der befragten Lehrkräfte im Bereich der auffälligen und weitere $11 \%$ im Feld der sehr auffälligen Erholungsunfähigkeit. Ferner gehören Personen in Dienstleistungsbe- 


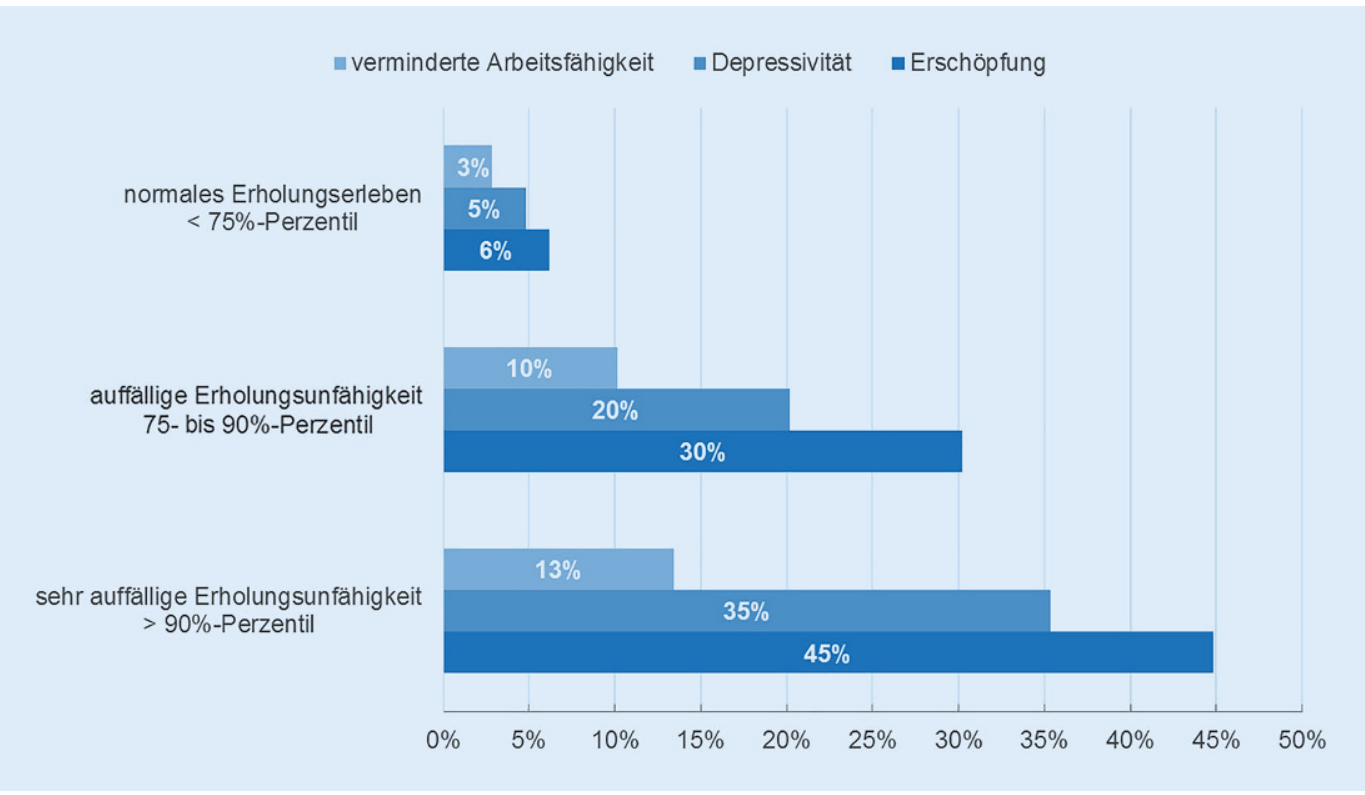

Abb. $3<$ Prozent der Beschäftigten mit verminderter Arbeitsfähigkeit, depressiver Symptomatik und Erschöpfung getrennt nach Graden der Erholung rufen dazu, von denen insgesamt $16 \%$ auffallende Erholungswerte erreichen. Gefolgt werden diese Erwerbstätigengruppen von Personen mit akademischen Berufen und Fachkräftetätigkeiten aus unterschiedlichen Bereichen wie der Informations- und Kommunikationstechnologie (14\%), dem Gesundheitswesen (14\%) oder der Betriebswirtschaft (13\%). Weniger betroffen sind Bediener von Anlagen und Maschinen sowie Personen in Montageberufen, von denen insgesamt $8 \%$ Auffälligkeiten beim Erholungserleben zeigen. Dies gilt auch für die Gruppe der Hilfsarbeitskräfte, von denen insgesamt $7 \%$ der Befragten im auffälligen und sehr auffälligen Wertebereich liegen. Eine detailliertere Darstellung der berechneten Werte befindet sich im Anhang (• Tab. 3).

\section{Arbeitsfähigkeit, mentale Gesundheit und Erholung}

Bei Betrachtung der unterschiedlichen Grade an Erholungseinschränkungen zeigt sich, dass mit ansteigendem Ausmaß der Erholungsprobleme gleichzeitig auch der Anteil an Personen mit gesundheitlichen Beeinträchtigungen zunimmt. So verfügen $3 \%$ von den im Normalbereich der Erholung liegenden Beschäftigten über eine verminderte Arbeitsfähigkeit (•Abb. 3). Dagegen sind es von den Beschäftigten mit auffälligen
Erholungsschwierigkeiten $10 \%$ und von denjenigen mit stark auffälligen Erholungseinschränkungen $13 \%$, die eine verminderte Arbeitsfähigkeit aufweisen.

Ein ähnliches Muster lässt sich auch für die Beziehung zwischen der Erholung und den beiden Indikatoren für die mentale Gesundheit feststellen (• Abb. 3). Unter den Beschäftigten im unauffälligen Bereich der Erholung befinden sich $5 \%$ mit depressiven und $6 \%$ mit Erschöpfungssymptomen. Hingegen zeigen von den Beschäftigten mit auffälligen Erholungseinschränkungen 20\% eine depressive Symptomatik und 30\% Erschöpfungsmerkmale. Unter den Befragten mit sehr auffälliger Erholungsunfähigkeit weisen $35 \%$ eine Depressionsund $45 \%$ eine Erschöpfungssymptomatik auf. Eine umfassendere Darstellung der berechneten Werte befindet sich im Anhang (• Tab. 4).

\section{Diskussion}

Die vorliegende Untersuchung gibt einen Überblick über die Verbreitung von Erholungsbeeinträchtigungen bei Beschäftigten in Deutschland, welche im Zuge der Digitalisierung stärker in den Fokus von Forschung und Praxis rücken. Die Analysen wurden mit Daten durchgeführt, die repräsentativ sind für in Deutschland sozialversicherungspflich- tig Beschäftigte im Alter von 31 bis 60 Jahren [12].

Die Ergebnisse der Untersuchung zeigen, dass sich der prozentuale Anteil an sozialversicherungspflichtig Beschäftigten mit Erholungsunfähigkeit auf insgesamt $13 \%$ beläuft. Dabei leiden $6 \%$ unter auffälligen und $7 \%$ unter stark auffälligen Erholungsschwierigkeiten, also andauernden Aktivierungszuständen nach dem Arbeitsende, Problemen mit dem mentalen Abschalten von der Arbeit sowie Schlafproblemen. Die Befragten sind hierbei unabhängig von Alter und Geschlecht prozentual ähnlich betroffen. Zusammen mit Befunden aus weiteren Studien, wonach Einzelaspekte der Erholungsunfähigkeit wie Probleme mit dem mentalen Abschalten in der Erwerbsbevölkerung weit verbreitet sind (43\% [13]; $38 \%$ [19]), unterstreichen die hier ermittelten Prävalenzraten die arbeitsmedizinische Bedeutung der Erholungsunfähigkeit.

Anhand der untersuchten Variablen wird deutlich, dass Erholungsprobleme mit bestimmten Beschäftigungsmerkmalen verbunden sind. Dazu gehören überlange Arbeitszeiten. Bei Arbeitszeiten von $48 \mathrm{~h}$ und mehr pro Woche sind die Erholungsmöglichkeiten während der Ruhezeit beschränkt - hier berichtet fast ein Drittel der Erwerbstätigen von Erholungsschwierigkeiten. Des Weiteren sind prozentual mehr Beschäftigte ohne 
feste Zeiten für deren Arbeitsbeginn und -ende von Erholungsproblemen betroffen, als Personen mit festen Arbeitszeiten, Gleitzeitregelungen oder Schichtarbeit.

Bei Betrachtung verschiedener Berufsgruppen befinden sich in der Gruppe der Führungskräfte anteilig am meisten Personen, die von Erholungsunfähigkeit betroffen sind. Diese tragen in der Regel zusätzliche Verantwortung, z. B. für Personal und/oder Finanzen, was eine adäquate Erholung erschweren kann. Auch Personen in Berufen mit interaktiven Tätigkeiten sind eher mit Erholungsproblemen konfrontiert, was vermutlich auf die damit einhergehenden emotionalen Arbeitsanforderungen zurückzuführen ist, die eine abträgliche Wirkung auf die Erholung haben können [17].

Erholungsbeeinträchtigungen selbst sind wiederum mit gesundheitsbezogenen Risiken verbunden. Mit steigendem Grad der berichteten Erholungsprobleme nimmt auch der Anteil an Personen mit Depressivität und Erschöpfung sowie verminderter Arbeitsfähigkeit zu. Diese Befunde verdeutlichen die Wichtigkeit der Beachtung von Erholungsproblemen bei Beschäftigten, um sowohl deren Gesundheit zu erhalten als auch deren Erwerbsteilhabe $\mathrm{zu}$ sichern. Nicht zuletzt ist eine verminderte Arbeitsfähigkeit prädiktiv für einen vorzeitigen Berufsausstieg [4].

\section{Limitationen und Ausblick}

In künftigen Studien sollten folgende Punkte zur Verbesserung ihrer Aussagekraft beachtet werden:

- Erweiterung der Altersspanne der Stichprobe auf jüngere (unter 31 Jahre) und ältere Personen (über 60 Jahre) sowie Selbstständige, freiberuflich Tätige und Beamte für ein vollumfängliches Bild der Prävalenz von Erholungsbeeinträchtigungen bei Beschäftigten;

- Betrachtung langfristiger Zusammenhänge mit Daten im Längsschnitt zur Untersuchung möglicher kausaler Wirkrichtungen zwischen Arbeit, Erholung und Gesundheit;

- Einbeziehung von Fremdbeobachtungsdaten (z. B. Depressions- diagnosen) zur Minimierung von Methodenverzerrungen aufgrund reiner Selbstauskünfte.

\section{Fazit für die Praxis}

\section{- Die Erfassung von Erholungsschwie- rigkeiten kann für die Erkennung weiterer Gesundheitsrisiken dienlich sein. \\ - Besonderes Augenmerk sollte dabei auf von Erholungsproblemen stärker betroffene Beschäftigtengruppen gelegt werden. Gemäß vorliegenden Ergebnissen sind dies v. a. Perso- nen mit Führungsverantwortung, häufiger Interaktionsarbeit, langen Arbeitszeiten sowie unbestimmten Zeiten für Arbeitsbeginn und -ende.}

\section{Korrespondenzadresse

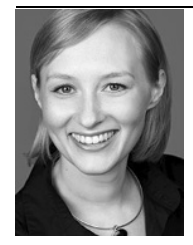 \\ Anika D. Schulz \\ FB 3 Arbeit und Gesundheit, Gruppe 3.4 Mentale Gesundheit und kognitive Leistungsfähigkeit, Bundesanstalt für Arbeitsschutz und Arbeitsmedizin Nöldnerstr. 40-42, 10317 Berlin, Deutschland schulz.anika2@baua.bund.de}

Förderung. Die Auswertungen wurden im Rahmen des von der Bundesanstalt für Arbeitsschutz und Arbeitsmedizin geförderten Projektes F 2431 „Erholung innerhalb und außerhalb des Arbeitskontextes - Wirkungen und Gestaltungsansätze im Wandel der Arbeit" durchgeführt.

\section{Einhaltung ethischer Richtlinien}

Interessenkonflikt. A.D. Schulz, J. Wendsche, A. Lohmann-Haislah und I. Schöllgen geben an, dass kein Interessenkonflikt besteht.

Für diesen Beitrag wurden von den Autoren keine Studien an Menschen oder Tieren durchgeführt. Für die aufgeführten Studien gelten die jeweils dort angegebenen ethischen Richtlinien. 


\section{Anhang}

Tab. 2 Beschäftigte getrennt nach Beschäftigungsmerkmalen mit unauffälligen, auffälligen und sehr auffälligen Einschränkungen in der Erholung Unauffälliges Erholungserleben Auffällige Erholungsunfähigkeit $\quad$ Sehr auffällige Erholungsunfähigkeit

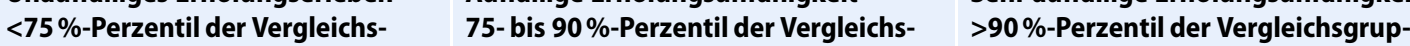
gruppe gruppe

pe

\begin{tabular}{|c|c|c|c|c|c|c|c|c|c|}
\hline & $N$ & $\%$ & $95 \%-K I$ & $N$ & $\%$ & $95 \%-K I$ & $N$ & $\%$ & $95 \%-K I$ \\
\hline \multicolumn{10}{|l|}{ Wochenarbeitszeit } \\
\hline$\leq 20 \mathrm{~h}$ & 493 & 96 & $93-97$ & 11 & 2 & $1-4$ & 15 & 2 & $1-4$ \\
\hline $21-30 \mathrm{~h}$ & 368 & 89 & $86-92$ & 23 & 6 & $4-9$ & 18 & 5 & $3-7$ \\
\hline $31-40 \mathrm{~h}$ & 1195 & 88 & $86-90$ & 73 & 6 & $4-7$ & 84 & 6 & $5-8$ \\
\hline $41-47 \mathrm{~h}$ & 836 & 86 & $84-88$ & 64 & 7 & $5-9$ & 69 & 7 & $6-9$ \\
\hline$\geq 48 \mathrm{~h}$ & 532 & 73 & $69-76$ & 80 & 11 & $9-14$ & 111 & 16 & $13-19$ \\
\hline \multicolumn{10}{|l|}{ Arbeitszeitlage $^{\mathrm{a}}$} \\
\hline $\begin{array}{l}\text { Keine feste Arbeits- } \\
\text { zeit }\end{array}$ & 475 & 80 & $77-84$ & 45 & 8 & $6-10$ & 74 & 12 & $9-15$ \\
\hline Feste Arbeitszeiten & 1588 & 87 & $85-89$ & 114 & 6 & $5-8$ & 118 & 7 & $5-8$ \\
\hline Schichtdienst & 2903 & 86 & $85-87$ & 208 & 6 & $6-7$ & 256 & 8 & $7-9$ \\
\hline Gleitzeit & 1038 & 88 & $86-90$ & 58 & 5 & $4-7$ & 80 & 7 & $5-9$ \\
\hline \multicolumn{10}{|l|}{ Vertragsart } \\
\hline Zeitlich unbefristet & 3285 & 86 & $85-88$ & 237 & 6 & $6-7$ & 281 & 7 & $6-8$ \\
\hline Zeitlich befristet & 200 & 84 & $78-88$ & 16 & 8 & $5-12$ & 18 & 9 & $5-14$ \\
\hline
\end{tabular}

Tab. 3 Berufsgruppen mit unauffälligen, auffälligen und sehr auffälligen Einschränkungen in der Erholung

Berufsgruppe
Unauffälliges Erholungserle-

ben

$<75 \%$-Perzentil der Vergleichsgruppe

N $\%$ (25)-KI
Auffällige Erholungsunfähigkeit

75- bis $90 \%$-Perzentil der Vergleichsgruppe
Sehr auffällige Erholungsunfähigkeit

$>90 \%$-Perzentil der Vergleichsgruppe

\begin{tabular}{|c|c|c|c|c|c|c|c|c|c|}
\hline & $N$ & $\%$ & $95 \%-K I$ & $N$ & $\%$ & $95 \%-K I$ & $N$ & $\%$ & $95 \%-K$ \\
\hline Führungskräfte & 190 & 72 & $66-78$ & 28 & 12 & $8-17$ & 39 & 16 & $11-21$ \\
\hline Lehrkräfte & 141 & 77 & $70-83$ & 18 & 12 & $7-18$ & 21 & 11 & $7-17$ \\
\hline $\begin{array}{l}\text { Juristen, Sozialwissenschaftler und Kul- } \\
\text { turberufe, juristische, sozialpflegerische, } \\
\text { kulturelle Fachkräfte }\end{array}$ & 151 & 81 & $74-87$ & 16 & 11 & $7-17$ & 15 & 8 & $5-13$ \\
\hline Dienstleistungsberufe und Verkäufer & 400 & 85 & $81-88$ & 31 & 7 & $5-10$ & 37 & 9 & $6-12$ \\
\hline $\begin{array}{l}\text { Akademische und vergleichbare Fach- } \\
\text { kräfte in der Informations- und Kommu- } \\
\text { nikationstechnologie }\end{array}$ & 100 & 85 & $77-91$ & 9 & 8 & $4-15$ & 6 & 6 & $3-13$ \\
\hline $\begin{array}{l}\text { Akademische und verwandte Gesund- } \\
\text { heitsberufe, Assistenzberufe im Gesund- } \\
\text { heitswesen }\end{array}$ & 316 & 86 & $82-89$ & 27 & 7 & $5-10$ & 27 & 7 & $5-10$ \\
\hline $\begin{array}{l}\text { Betriebswirte und vergleichbare akade- } \\
\text { mische Berufe, betriebswirtschaftliche } \\
\text { und kaufmännische Fachkräfte }\end{array}$ & 481 & 87 & $83-89$ & 28 & 5 & $3-7$ & 46 & 8 & $6-11$ \\
\hline $\begin{array}{l}\text { Naturwissenschaftler, Mathematiker und } \\
\text { Ingenieure, ingenieurtechnische und } \\
\text { vergleichbare Fachkräfte }\end{array}$ & 300 & 88 & $84-91$ & 22 & 6 & $4-9$ & 20 & 6 & $4-9$ \\
\hline $\begin{array}{l}\text { Fachkräfte in Land- und Forstwirtschaft } \\
\text { und Fischerei, Handwerks- und ver- } \\
\text { wandte Berufe }\end{array}$ & 530 & 88 & $85-91$ & 34 & 6 & $4-8$ & 37 & 6 & $4-9$ \\
\hline Bürokräfte und verwandte Berufe & 453 & 88 & $85-91$ & 26 & 6 & $4-8$ & 33 & 6 & $4-9$ \\
\hline
\end{tabular}




\begin{tabular}{|c|c|c|c|c|c|c|c|c|c|}
\hline \multirow[t]{2}{*}{ Berufsgruppe } & \multicolumn{3}{|c|}{$\begin{array}{l}\text { Unauffälliges Erholungserle- } \\
\text { ben } \\
<75 \% \text {-Perzentil der Ver- } \\
\text { gleichsgruppe }\end{array}$} & \multicolumn{3}{|c|}{$\begin{array}{l}\text { Auffällige Erholungsunfähig- } \\
\text { keit } \\
\text { 75- bis } 90 \% \text {-Perzentil der Ver- } \\
\text { gleichsgruppe }\end{array}$} & \multicolumn{3}{|c|}{ 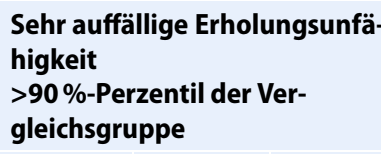 } \\
\hline & $N$ & $\%$ & $95 \%-K I$ & $N$ & $\%$ & $95 \%-K I$ & $N$ & $\%$ & $95 \%-K I$ \\
\hline $\begin{array}{l}\text { Bediener von Anlagen und Maschinen, } \\
\text { Montageberufe }\end{array}$ & 264 & 91 & $88-94$ & 14 & 4 & $3-7$ & 13 & 4 & $2-7$ \\
\hline Hilfsarbeitskräfte & 260 & 93 & $89-96$ & 7 & 3 & $2-7$ & 12 & 4 & $2-7$ \\
\hline
\end{tabular}

Tab.4 Beschäftigte mit schlechter Arbeitsfähigkeit, depressiver Symptomatik und Erschöpfung getrennt nach unauffälligen, auffälligen und sehr auffälligen Einschränkungen in der Erholung

\begin{tabular}{|c|c|c|c|c|c|c|c|c|c|}
\hline & \multicolumn{3}{|c|}{$\begin{array}{l}\text { Unauffälliges Erholungserleben } \\
<75 \% \text {-Perzentil der Vergleichsgrup- } \\
\text { pe }\end{array}$} & \multicolumn{3}{|c|}{$\begin{array}{l}\text { Auffällige Erholungsunfähigkeit } \\
75 \text { - bis } 90 \% \text {-Perzentil der Vergleichs- } \\
\text { gruppe }\end{array}$} & \multicolumn{3}{|c|}{$\begin{array}{l}\text { Sehr auffällige Erholungsunfähigkeit } \\
>90 \% \text {-Perzentil der Vergleichsgruppe }\end{array}$} \\
\hline & $N$ & $\%$ & $95 \%-K I$ & $N$ & $\%$ & $95 \%-K I$ & $N$ & $\%$ & $95 \%-K I$ \\
\hline $\begin{array}{l}\text { Verminderte } \\
\text { Arbeitsfähig- } \\
\text { keit }\end{array}$ & 102 & 3 & $2-3$ & 25 & 10 & $7-15$ & 40 & 13 & $10-18$ \\
\hline Depressivität & 145 & 5 & $4-6$ & 43 & 20 & $15-26$ & 99 & 35 & $30-41$ \\
\hline Erschöpfung & 197 & 6 & $5-7$ & 71 & 30 & $24-37$ & 129 & 45 & $39-51$ \\
\hline
\end{tabular}

Open Access. Dieser Artikel wird unter der Creative Commons Namensnennung 4.0 International Lizenz (http://creativecommons.org/licenses/by/4.0/deed de) veröffentlicht, welche die Nutzung, Vervielfältigung, Bearbeitung, Verbreitung und Wiedergabe in jeglichem Medium und Format erlaubt, sofern Sie den/die ursprünglichen Autor(en) und die Quelle ordnungsgemäßnennen, einen Linkzur Creative Commons Lizenz beifügen und angeben, ob Änderungen vorgenommen wurden.

\section{Literatur}

1. Demerouti E (1999) Burnout: Eine Folge konkreter Arbeitsbedingungen bei Dienstleistungs-und Produktionstätigkeiten. Peter Lang, Frankfurt am Main

2. Demerouti E, Mostert K, Bakker AB (2010) Burnout and work engagement: $A$ thorough investigation of the independency of both constructs. J Occup Health Psychol 15:209-222

3. Geurts SA, Sonnentag S (2006) Recovery as an explanatory mechanism in the relation between acute stress reactions and chronic health impairment. Scand J Work Environ Health 32:482-492

4. Hasselhorn HM, Freude G (2007) Der Work Ability Index - ein Leitfaden. Wirtschaftsverlag NW, Bremerhaven

5. Löwe B, Spitzer R, Zipfel S et al (2002) Gesundheitsfragebogen für Patienten (PHQ D). Komplettversion und Kurzform. Testmappe mit Manual, Fragebögen, Schablonen. Pfizer, Karlsruhe

6. International Labour Organization (2008) International standard classification of occupations: ISCO-08. In: Structure, group definitions and correspondence tables. International Labour Office. ILO Geneva
7. Rau R (2004) Job strain or healthy work: a question of task design. JOccup Health Psychol 9:322-338

8. Rau R (2011) Zur Wechselwirkung von Arbeit, Beanspruchung und Erholung. In: Bamberg $E$, Ducki A, Metz A-M (Hrsg) Gesundheitsförderung und Gesundheitsmanagement in der Arbeitswelt: Ein Handbuch. Hogrefe, Göttingen, S847-847

9. Richter P, Funke C, Mittmann S et al (2017) Gesundheitsrelevante Beeinflussung der Handlungsregulation unter psychischer Belastung - Entwicklung von Parallelskalen zum FABAFragebogen. Psychologie des Alltagshandelns 10:5-18

10. Richter P, Rudolf M, Schmidt CF (1996) Fragebogen zur Analyse belastungsrelevanter Anforderungsbewältigung (FABA) - Handanweisung und Fragebogen. Swets, Frankfurtam Main

11. Richter P, Rudolf M, Schmidt CF (1999) Fragebogen zur Analyse belastungsrelevanter Anforderungsbewältigung: FABA. Swets \& Zeitlinger, Frankfurt am Main

12. Rose U, Schiel S, Schröder H et al (2017) The study on mental health at work: design and sampling. Scand J Public Health 45:584-594

13. Seiler K, Beerheide E, Figgen M, Goedicke A, Alaze F, Rack R, Mayer S, Loocke-Scholz A, Evers G (2013) Arbeit, Leben und Erholung - Ergebnisse einer Repräsentativbefragung in Nordrhein-Westfalen. Landesinstitut für Arbeitsgestaltung des Landes Nordrhein-Westfalen, Düsseldorf

14. Spitzer RL, Kroenke K, Williams JB (1999) Validation and utility of a self-report version of PRIME-MD: the $\mathrm{PHQ}$ primary care study. Primary care evaluation of mental disorders. Patient health questionnaire. JAMA 282:1737-1744

15. Tuomi K, Ilmarinen J, Jahkola A et al (2003) Arbeitsbewältigungsindex. Wirtschaftsverlag NW, Bremerhaven

16. Tuomi K, Ilmarinen J, Jahkola A et al (1998) Work ability index. Finnish Institute of Occupational Health, Helsinki
17. Wendsche J, Lohmann-Haislah A (2017) A meta-analysis on antecedents and outcomes of detachment from work. Front Psychol 7:1-24

18. Wieland-Eckelmann R, Baggen R (1994) Beanspruchung und Erholung im Arbeits-Erholungs-Zyklus. In: Wieland-Eckelmann R, Allmer H, Kallus KW, Otto JH (Hrsg) Erholungsforschung. Beiträge der Emotionspsychologie, Sportpsychologie und Arbeitspsychologie. Psychologie Verlagsunion, Weinheim, S 102-154

19. Wohlers K, Hombrecher M (2016) Entspann dich Deutschland: Die TK-Stressstudie 2016. Techniker Krankenkasse, Hamburg 